\title{
Tumor Cells Are the Dip on This Chip
}

\section{Stephan C. Jahn, Ph.D.}

Department of Medicinal Chemistry and UF Health Cancer Center, University of Florida, Gainesville, FL

Email: scjahn@ufl.edu

\begin{abstract}
In order to improve patient treatment, scientists are always searching for better, faster, and cheaper ways of doing things. Microfluidic chips are becoming increasingly popular in medicine due to their advanced capabilities and low cost. In this research highlight, we look at a paper by Peterson et al. in which they develop a microfluidic method utilizing ascites fluid from ovarian cancer patients that can very sensitively detect and characterize the tumor cells present in the fluid oftentimes drained from these patients. The technology has numerous possibilities in clinical oncology and beyond.
\end{abstract}

\section{Introduction}

It is well accepted that the sooner a cancer patient is diagnosed, the greater the chances of a positive treatment outcome. While our cancer detection capabilities have improved greatly, there is still a long way to go. Once the tumor is detected, characterization of it generally requires a biopsy. These tend to be quite painful for the patient, limiting the number of times it can be completed, and do not adequately address tumor heterogeneity in a large tumor. In a recent issue of PNAS, Peterson et al. present a technique in which they are able to isolate, purify, and analyze ascites tumor cells (ATCS), ovarian cancer cells from peritoneal fluid, using an inexpensive microfluidic chip [1]. This ascites fluid is routinely drained from ovarian cancer patients, providing a ready supply of cells that can be processed at the point of care. This technology will undoubtedly improve the detection, characterization, and monitoring of ovarian cancer patients as well as lead to many other uses in the health care industry.

\section{Authors' Results}

The researchers first set out to identify a protein signature that could be detected by commercially available antibodies to distinguish cancer cells from normal cells. After identifying potential proteins, the antibodies were screened against a panel of cancer and normal cell lines. Those antibodies that did not provide a false positive or negative were then tested against ascites fluid collected from both cancer and noncancer patients. It was determined that epithelial cell adhesion molecule (EpCAM), CD24, and tumor-associated glycoprotein 72 (TAG-72) served as the best markers for cancer cells, while D2-40 and thrombomodulin were the best mesothelial, non-cancer markers. A cancer signature of positive EpCAM expression and/or vimentin expression while also lacking calretinin and CD45 was able to correctly classify all 65 patients tested using a flow cytometry method. The collected fluid had an average of 120,000 cells $/ \mathrm{mL}$ with a mean of 27,000 tumor cells $/ \mathrm{mL}$ in the cancer patients.

The next step was translating this process into an inexpensive point of care system. The microfluidic chip consists of a series of microwells facing upstream to the sample flow. These wells are only large enough to hold one cell yet contain a small hole that allows small normal cells to pass through while catching the relatively larger cancer cells (illustrated in Figure 1). Prior to passing through the chip, samples are treated with primary and secondary antibodies that will be used for cell analysis upon processing. Additionally, CD45 positive cells are removed magnetically and clumps are removed by a miniature $70 \mu \mathrm{m}$ filter. These purification steps yielded cancer cells that were approximately 1,000 fold more concentrated than in the ascites fluid. Once captured, the cells 
can easily be imaged using standard fluorescent microscopy (Figure 2).

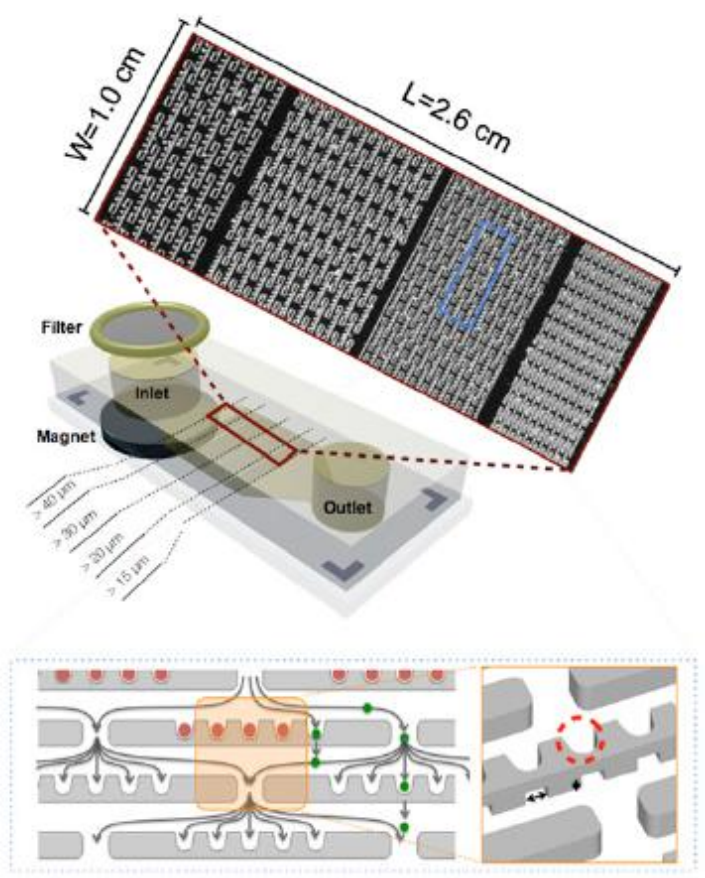

Figure 1. Illustration of the microfluidic chip. Cells enter through the inlet before passing through the body of the chip where they are caught in microwells (bottom left). Relatively smaller normal cells pass through escape holes in the wells (bottom right) making the chip selective toward cancer cells. Image courtesy of R. Weissleder.

This chip offers a number of advantages to the flow cytometry method. It needs only $100 \mu \mathrm{L}$ of sample for even the samples with few tumor cells while flow cytometry would require upwards of a half-liter. It is manufactured with polydimethylsiloxane and costs less than \$1 each. The authors were able to recollect the captured cells and use them for other assays such as mRNA measurement.

The chip was tested on an ovarian cancer patient undergoing treatment as a way to monitor treatment success. Upon initial administration of a cytotoxic regimen, the patient improved clinically while the ATC count decreased. An increase in ATCs detected correlated with patient relapse and then they decreased upon a subsequent positive response to antiangiogenic therapy. Additionally, by utilizing a larger set of protein markers on ATCS collected on the chip, the authors were able to differentiate tumors that responded well to treatment from those that were refractory.

This new technology will certainly find its way into the clinic. It has the ability to detect cancer cells in an undiagnosed patient, serve as a biopsy without additional patient discomfort, can serve as a non-harmful measure of tumor burden, determine the likelihood of treatment success, and provide an invaluable tool for doctors in areas of the world that do not have large financial or technical resources for cancer care. It will be exciting to see what additional uses for this unique system can be found.

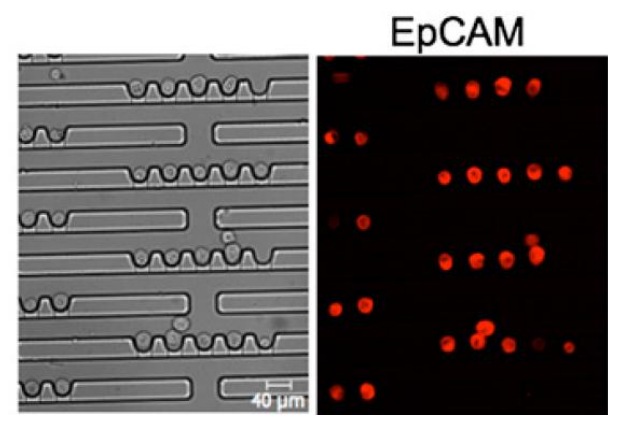

Figure 2. Representative images of cells on a microfluidic chip. Light (left) and fluorescent (right) microscopy images of ATCs captured in the chip's microwells. Image courtesy of $R$. Weissleder.

\section{Acknowledgements}

I thank Dr. Ralph Weissleder for his permission to highlight this work as well as the use of the original figures and the reviewers for their helpful critiques.

\section{References}

1. Peterson VM, Castro CM, Chung J, Miller NC, Ullal AV, Castano MD, Penson RT, Lee $H$, Birrer MJ, Weissleder R (2013) Ascites analysis by a microfluidic chip allows tumorcell profiling. PNAS 110(51):E4978-86. 